\title{
Experimental study of effect of stenosis geometry on pressure loss for periodic flow
}

\author{
Ondřej Veselý 1 ,a, Ludmila Nováková ${ }^{1}$ and Josef Adamec ${ }^{1}$ \\ ${ }^{1}$ CTU in Prague, Faculty of Mechanical Engineering, Department of Fluid Dynamics and Thermodynamics, Technická 4, 16607, Prague 6, \\ Czech Republic
}

\begin{abstract}
A stenosis is a narrowing in a tubular organ. In medicine, vessel stenosis poses health risk for people. In the last work, experimental investigation of pressure loss coefficient for varying stenosis eccentricity and shape for steady flow were performed. In this work, experimental investigation of pressure loss for varying stenosis eccentricity and shape under periodic flow were performed. Four models of different geometry were studied, two models are axisymmetric stenoses and two models are eccentric stenoses. All models were stenosis of $75 \%$ area reduction. The periodic flow, generated by a controllable pump, has sinus shape in an inlet. The measuring range of medium Reynolds number was from 500 to 1500 , range of ratio between an amplitude and medium flow rate was from 0.2 to 0.6 and range of frequency was from 0.2 to $1 \mathrm{~Hz}$. The pressure loss for each conditions was quantified by mean value, amplitude and phase shift against flow rate.
\end{abstract}

\section{Introduction}

Stenosis is a narrowing of vessel. Stenosis significantly affect the blood flow, which has implication for the body system. [1] The characteristic feature of the stenoses is atherosclerosis [2].

The relationship between flow parameters and occurrence of the atherosclerosis has been studied by many studies [3], [4], [5]. The complexity of this issue, high demands on measurement accuracy and ethical limitations transfer research from the medical field to the field of technology. In laboratory for measuring flow parameters is used technically advanced method (for example PIV).

In fluid dynamics, the blood flow is an unsteady flow of the non-Newtonian fluid through an elastic tube. For certain experiments conditions the non-Newtonian characteristics can be neglected and the use of Newtonian fluid is sufficient [6], [7]. Many research is focused on models with a solid [8], [9] as well as elastic wall [10], [11]. This work is focused on model with solid wall.

The effect of unsteadiness is described in several experimental and numeric work. For example Siouffi et al. [12] compared different types of pulsatile flows with physiological one. This experimental work is focused on effect of stenosis geometry on pressure loss for unsteady flow, which have sinus shape in an inlet.

For the description of unsteady flow is used a dimensionless number to be called the Womersley number. It is an expression of the pulsatile flow frequency in relation to viscous effect. The Womersley number is defined by the relation:

$$
\alpha=r \sqrt{\frac{\omega}{v}}
$$

where $r$ is the diameter of unobstructed vessel and $v$ is the kinematic viscosity.

In this work four models were studied; two symmetric stenoses and two eccentric stenoses. All models have circular cross-section and they were stenosis with $75 \%$ area reduction. The measuring range of Womersley number was from 8 to 18 .

From obtained data, the local pressure loss is evaluated from equation:

$$
\Delta p_{l}=\Delta p_{t}-\Delta p_{f}
$$

where $\Delta \mathrm{p}_{\mathrm{t}}$ is total pressure loss and $\Delta \mathrm{p}_{\mathrm{f}}$ is major pressure loss. The total pressure loss is sum of major and local pressure loss. From obtained data amplitude, mean value and phase shift of local pressure loss was evaluated. Amplitude of local pressure loss is given by equation:

$$
\Delta p_{a, l}=\frac{\Delta p_{\max , l}-\Delta p_{\min , l}}{2}
$$

where $\Delta \mathrm{p}_{\max , 1}$ is maximum of the local pressure loss and $\Delta \mathrm{p}_{\text {min,l }}$ is minimum of the local pressure loss. Mean value is given by equation.

$$
\Delta p_{m, l}=\frac{\Delta p_{\max , l}+\Delta p_{\min , l}}{2}
$$

Phase shift of local pressure loss is given by equation:

\footnotetext{
${ }^{\mathrm{a}}$ Corresponding author: O.Vesely@fs.cvut.cz
} 


$$
\Delta \varphi_{l}=\varphi_{\max , f r}-\varphi_{\max , l},
$$

where $\varphi_{\text {max,fr }}$ is phase of maximum value of flow rate and $\varphi_{\text {max }, 1}$ is phase of maximum value of local pressure loss.

The geometry of stenosis models is shown in Figure 1, 2, 3 and 4. All models have the diameter of unobstructed cross-section $14 \mathrm{~mm}$, the diameter of obstructed crosssection $7 \mathrm{~mm}$, the length of model $50 \mathrm{~mm}$, the length of stenosis $5 \mathrm{~mm}$.

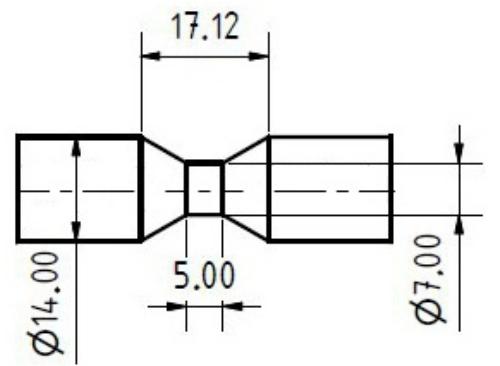

Figure 1. Symmetric stenosis number 1, narrowing angle $45^{\circ}$.

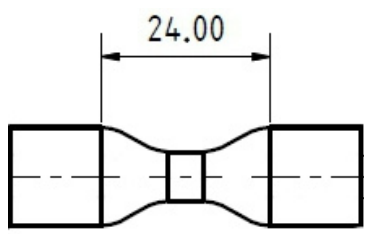

Figure 2. Symmetric stenosis number 2, smooth transition portion.

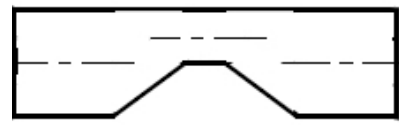

Figure 3. Eccentric stenosis number 1, narrowing angle $45^{\circ}$.

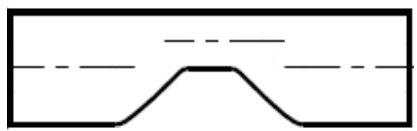

Figure 4. Eccentric stenosis number 2, smooth transition portion

The aim of this experimental study is determine, if pressure loss was affected more by shape of stenosis or by eccentricity.

\section{Method}

Schematic diagram of the experimental setup for measuring the pressure loss is shown in Figure 5.

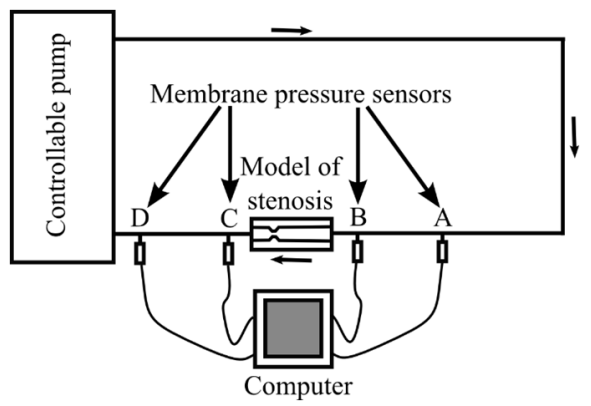

Figure 5. Experimental setup.
The unsteady flow was established in the model. The pressure loss was measured by four membrane pressure sensors. The distance between sensors is $70 \mathrm{~cm}$. The sensors were calibrated before measurement using water column.

For each flow regime the pressure gradient between sensors was recorded. Pressure gradient between sensor A and sensor B (sensors are labelled in Figure 5) is major pressure loss in pipe with length $70 \mathrm{~cm}$.

Between sensor B and C was measured total pressure loss, which is combination of major and local pressure loss. Sensor D is used to check the correct functioning of the sensors. The control is performed by comparison of data obtained between sensor A and B and between sensor $\mathrm{C}$ and D.

\section{Results}

Table 1 summarises the set of experimental flow regime. For each flow regime, measurement for five value of frequency was performed.

Table 1. Flow regimes

\begin{tabular}{|c|c|c|c|}
\hline $\begin{array}{l}\text { Number of } \\
\text { regime }\end{array}$ & $\begin{array}{c}\text { Mean } \\
\text { Reynolds } \\
\text { number } \\
\text { (1) }\end{array}$ & $\begin{array}{c}\text { Amplitude } \\
\text { of } \\
\text { Reynolds } \\
\text { number } \\
\text { (1) }\end{array}$ & $\begin{array}{c}\text { Range of } \\
\text { measured } \\
\text { frequency } \\
(\mathrm{Hz})\end{array}$ \\
\hline 1 & 500 & 100 & \multirow{9}{*}{$0.2-1.0$} \\
\hline 2 & 500 & 200 & \\
\hline 3 & 500 & 300 & \\
\hline 4 & 1000 & 200 & \\
\hline 5 & 1000 & 400 & \\
\hline 6 & 1000 & 600 & \\
\hline 7 & 1500 & 300 & \\
\hline 8 & 1500 & 600 & \\
\hline 9 & 1500 & 900 & \\
\hline
\end{tabular}

Figures $6-8$ plot results for symmetric stenosis number 1 . The results show that the difference of maximum and minimum value of mean and amplitude local pressure loss increases with increasing Reynolds number. The value of phase shift increase with Reynolds number and maximum value has for $\mathrm{Re}=1500$.

Figures $9-11$ plot results for symmetric stenosis number 2. This stenosis has smoother transition portion then symmetric stenosis number 1 . The results show that the value of mean and amplitude of local pressure loss is approximately same with stenosis number 1 .

Figures 12 - 14 plot results for eccentric stenosis number 1 . Comparing with symmetric stenosis number 1 , the eccentric stenosis has higher difference of maximum and minimum value of mean local and amplitude pressure loss. The value of phase shift increase with Reynolds number and maximum value has for $\mathrm{Re}=1000$ and 1500 .

Figures $15-17$ plot results for eccentric stenosis number 2. This stenosis has smoother transition portion then eccentric stenosis number 1 . The results show that this stenosis has lower the difference of maximum and minimum value of mean and amplitude local pressure loss 
is lower than in the eccentric stenosis number 1 . The phase shift for $\mathrm{Re}=500$ is lower than eccentric stenosis number 1 , for higher Re the value is same.

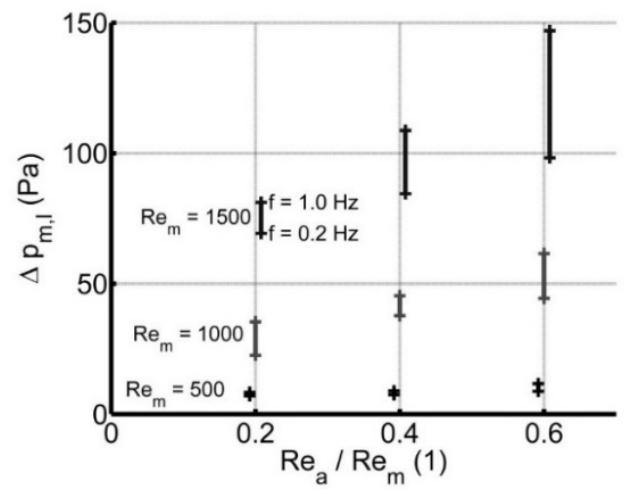

Figure 6. The mean value of local pressure loss for symmetric stenosis number 1.

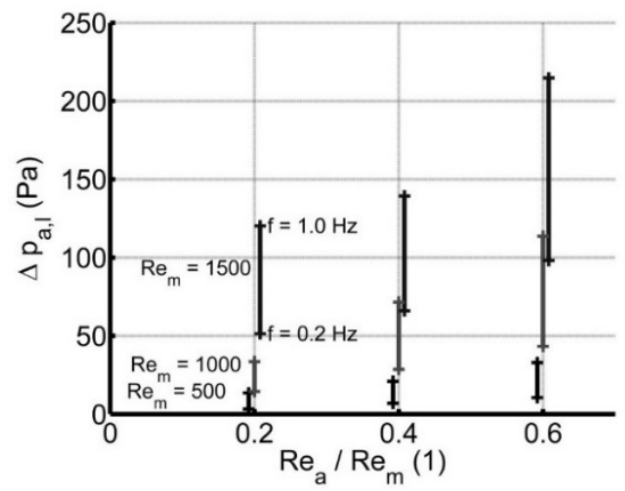

Figure 7. The amplitude of local pressure loss for symmetric stenosis number 1 .

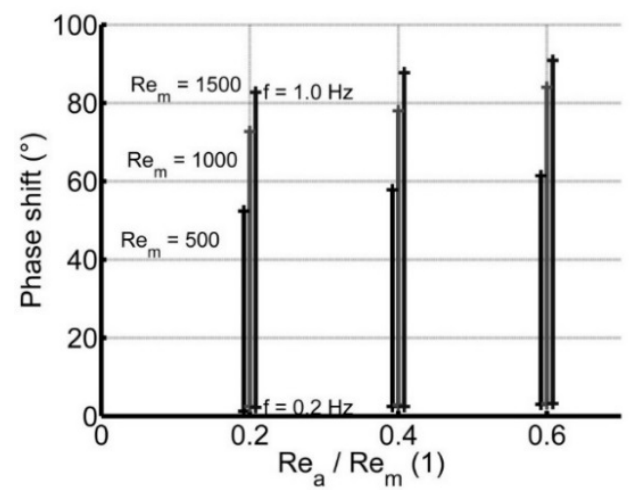

Figure 8. The phase shift of local pressure loss for symmetric stenosis number 1 .

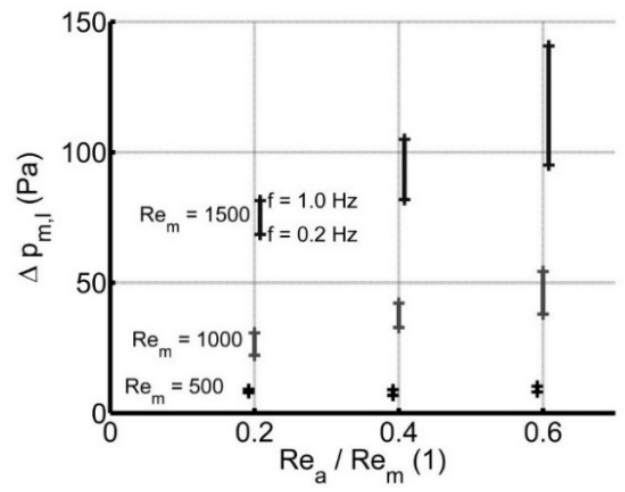

Figure 9. The mean value of local pressure loss for symmetric stenosis number 2 .

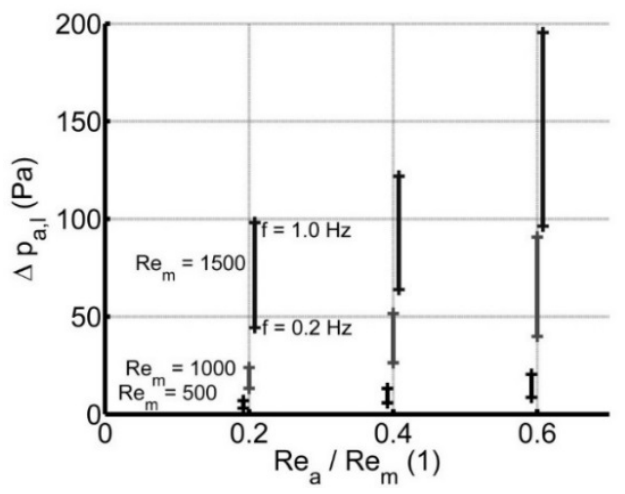

Figure 10. The amplitude of local pressure loss for symmetric stenosis number 2 .

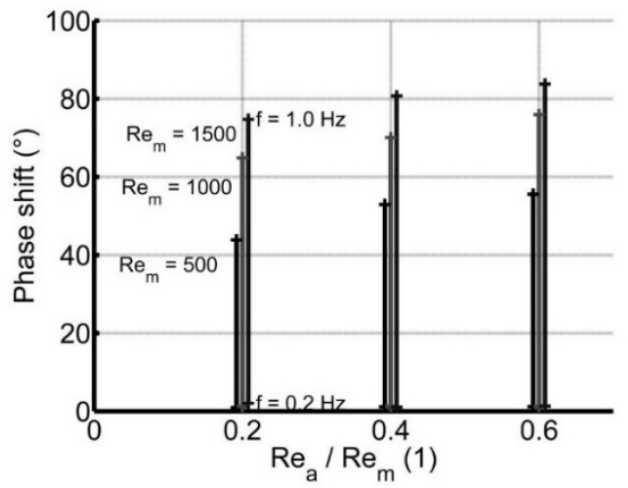

Figure 11. The phase shift of local pressure loss for symmetric stenosis number 2 .

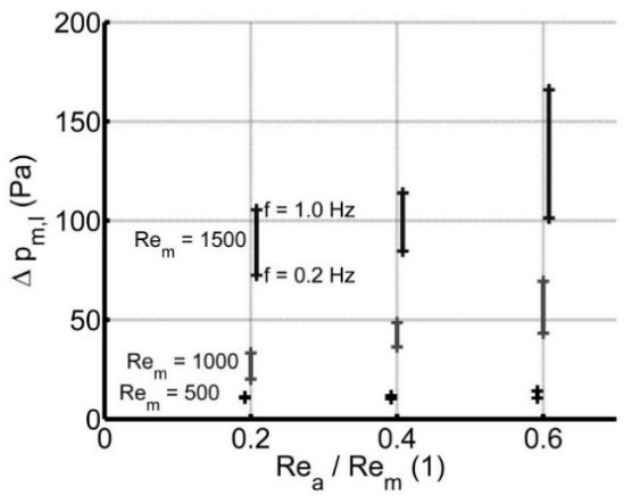

Figure 12. The mean value of local pressure loss for eccentric stenosis number 1 .

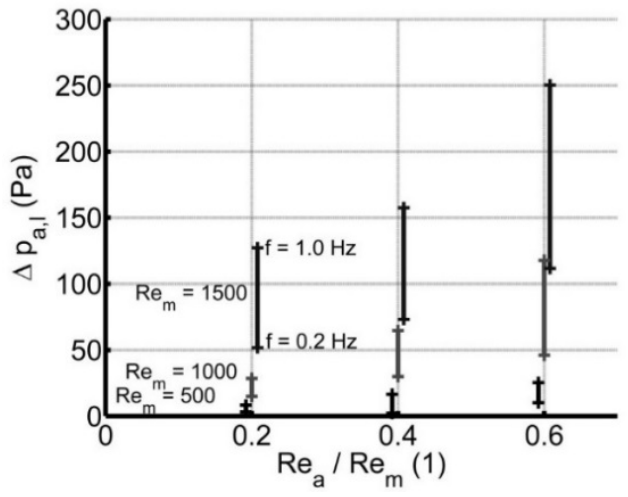

Figure 13. The amplitude of local pressure loss for eccentric stenosis number 1 . 


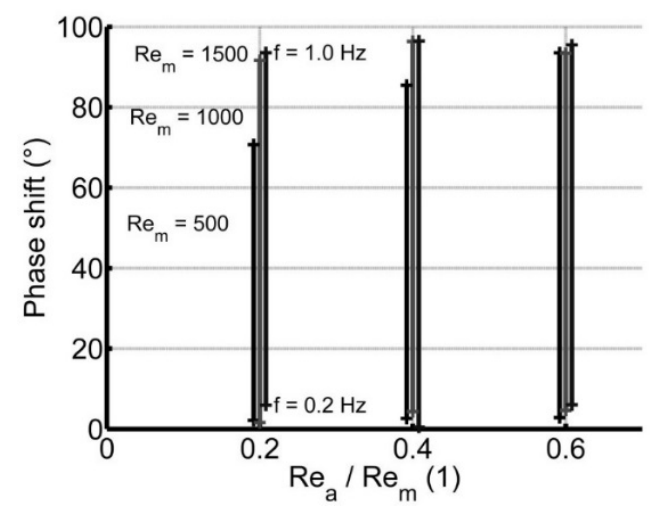

Figure 14. The phase shift of local pressure loss for eccentric stenosis number 1 .

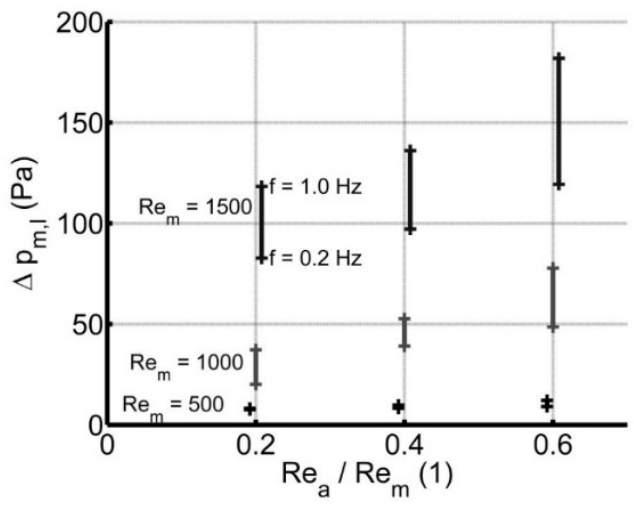

Figure 15. The mean value of local pressure loss for eccentric stenosis number 2.

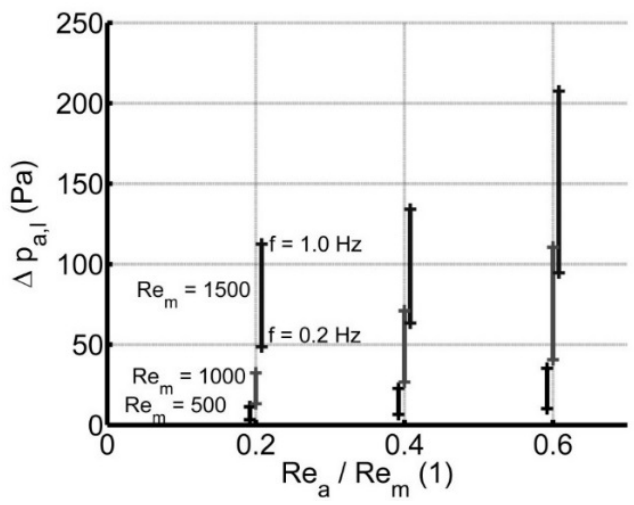

Figure 16. The amplitude of local pressure loss for eccentric stenosis number 2 .

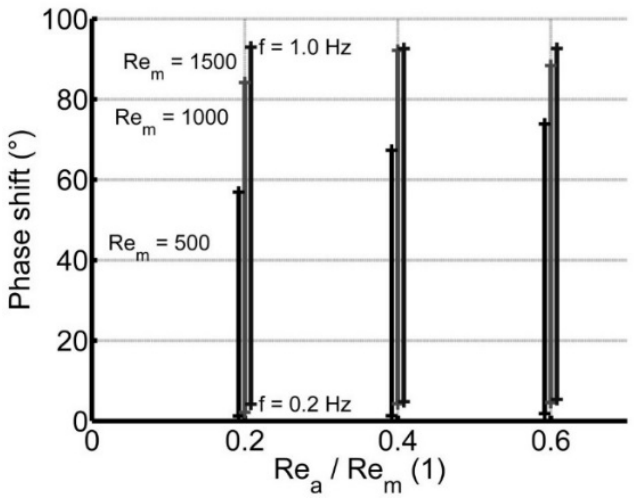

Figure 17. The phase shift of local pressure loss for eccentric stenosis number 2 .

\section{Conclusion}

In this work, experimental investigation of pressure loss of stenosis for the four models were performed: two symmetric stenoses and two eccentric stenoses. All models were stenosis of $75 \%$ area reduction. The results prove the relationship between pressure loss and varying eccentricity and shape of stenosis.

For all models, difference of maximum and minimum value of mean and amplitude local pressure loss increases with increasing Reynolds number. The eccentric stenoses have higher value of difference than symmetric stenoses. The phase shift for $\mathrm{Re}=500$ is higher for eccentric stenoses. The symmetric stenoses have maximum value of phase shift in $\mathrm{Re}=1500$, the eccentric stenoses in $\mathrm{Re}=$ 1000. The maximum value is approximately same for all models.

Investigation of the local pressure loss indicates that eccentric stenoses create higher pressure loss and higher difference between maximum and minimum value. The loss is little affected by shape of stenosis, but significantly by the eccentricity.

\section{References}

1. L. Caplain, J Am Med Association, 300, 81 - 90 (2008)

2. A. Malek, S. Alper, S. Izumo, J Am Med Association, 282, 2035 - 2042 (1999)

3. S. Glagov, C. K. Zarins, D. P. Giddens, D. N. Ku, Arch. Pathol. Lab. Med., 316, 1371 - 1375 (1988)

4. Y. Jiang, K. Kohara, K. Hiwada, Am Jounar of Hypertension, 13, $892-898$ (2000)

5. C. G. Caro, Arteriscler Thromb Vasc Biol., 29, $158-$ $161(2009)$

6. T. M. Liou, S. N. Liou, In Proc. Natl. Sci. Counc. ROC(B), 23, 133 - 148 (1999)

7. D. N. Ku, Ann Rev Fluid Mech., 29, 399 - 434 (1997)

8. A. S. Shuib, P. R. Hoskins, W. J. Easson, Engineering and Technology, 60, $56-69$ (2011)

9. B. Liu, Medical Engineering and Physics, 29, 868 876 (2007)

10. K. Pielhop, M. Klaas, W. Scroder, European Journal of Mechanics of Fluid, 35, $102-110$ (2012)

11. J. Kolínský, J. Matěcha, J. Novotný, In Proc. Of. ASME 2009 Fluids Engineering Division, New York, (2009)

12. M. Siouffi, V. Deplano, R. Pélisser, Journal of Biomechanics, 31, 11 - 19 (1998)

\section{Acknowledgement}

The research has been supported by Faculty of Mechanical Engineering of the Czech Technical University in Prague through the student grant project No. SGS15/191/OHK2/3T/12 (The Unsteady flow in Pipe with Variable Area Cross Section). 